\title{
A two-step species-specific 16S rRNA PCR assay for the detection of Taylorella equigenitalis in horses
}

\begin{abstract}
Thomas C. Buckley ${ }^{1}$, B. Cherie. Millar ${ }^{2}$, Claire L. Egan ${ }^{1}$, Paula Gibson ${ }^{2}$, Hazel Cosgrove ${ }^{1}$, Siobhan Stanbridge ${ }^{1}$,
\end{abstract} Motoo Matsuda ${ }^{3}$ and John E. Moore ${ }^{2}$

1 Irish Equine Centre, Johnstown, Naas, Co Kildare, Republic of Ireland.

2 Northern Ireland Public Health Laboratory, Department of Bacteriology, Belfast City Hospital, Belfast BT9 7AD, Northern Ireland.

3 Laboratory of Molecular Biology, School of Environmental Health Sciences, Azabu University, 1-17-71 Fuchinobe, Sagamihara,

229, Japan.

A two-step PCR assay was developed for the molecular detection of Taylorella equigenitalis, a Gram-negative genital bacterial pathogen in horses. Two specific oligonucleotide primers (TE16SrRNABCHf [25mer] and TE16SrRNABCHr [29mer]) were designed from multiple alignments of the $16 \mathrm{~S}$ rRNA gene loci of several closely related taxa, including $\mathrm{T}$. asinigenitalis. Subsequent enhanced surveillance of 250 Thoroughbred animals failed to detect the presence of this organism directly from clinical swabs taken from the genital tract of mares and stallions. Such a molecular approach offers a sensitive and specific alternative to conventional culture techniques, and has the potential to lead to improved diagnosis and subsequent management of horses involved in breeding programmes.

\author{
Key words \\ Horse, \\ Taylorella equigenitalis, \\ $16 \mathrm{~S}$ rRNA, \\ Contagious equine \\ metritis, \\ Polymerase chain reaction.
}

\section{Introduction}

Contagious equine metritis (CEM) caused by Taylorella equigenitalis is a sexually transmitted disease in horses, generally leading to a loss of fertility in the mare. Since CEM was first reported in England (Crowhurst, 1977), this disease and its causative agent have been detected in many countries and in various breeds of horses (Timoney, 1996). Diagnosis of CEM has been based mainly on the isolation of T. equigenitalis by culture, from swabs of the central clitoral sinus in mares and from the urethral fossa in stallions. Previously, a three-step PCR assay based on the amplification of the 16S rRNA of Taylorella equigenitalis was described by Bleumink-Pluym et al. (1994), yielding a 585bp amplicon specific to T. equigenitalis. However, through careful re-design of primers, it is possible to modify the assay conditions, by eliminating the annealing time during the PCR reaction. Therefore, it was the aim of this study to

Corresponding author:

Thomas C. Buckley,

Irish Equine Centre,

Johnstown, Naas,

Co. Kildare,

Ireland.

Tel: +35345866266

Fax: +35345866273

E-mail: iec@equine-centre.ie develop a PCR diagnostic assay specific for T. equigenitalis, based on 16S rRNA, in a two-step amplification procedure, thereby reducing assay time and hence allowing for more rapid laboratory turnaround times to aid with the management of animals being screened for this organism. The two-step assay was used to survey 250 Thoroughbred horses for the presence of this organism.

\section{Materials and methods}

Primer design

Alignments of the $16 \mathrm{~S}$ rRNA gene sequence of Taylorella equigenitalis [GenBank Accession no: X68645] was performed against 23 bacterial species of close phylogenetic relatedness, as previously described (Bleumink-Pluym et al., 1994 ), employing MegAlign software (Dnastar Inc, Wisconsin, USA), as well as with Alcaligenes xylosoxidans, Bordetella bronchiseptica, Haemophilus parainfluenzae, $H$. influenzae, $H$. ducreyi, $H$. parasuis, $H$. aphrophilus, $H$. parahaemolyticus, $H$. paraphrophi lus, H. segnis, $H$. haemoglobinophilus, $H$. paracuniculus, $H$. paragallinarum, $H$. taxon C., $H$. haemolyticus, $H$. somnus, $H$. aegyptius, $H$. haemophilus paraphrobaemolyticus and Taylorella asinigenitalis. Primers were designed to allow for a two-step PCR amplification with a high melting temperature (Tm), as described in Figure 1. 


\section{DNA extraction from bacterial organisms}

All DNA isolation procedures were carried out in a Class II Biological Safety Cabinet (MicroFlow, England) in a room physically separated from that used to set up nucleic acid amplification reaction mixes and also from the "post-PCR" room in accordance with the Good Molecular Diagnostic Procedures (GMDP) guidelines of Millar et al. (2002), in order to minimise contamination and, hence, the possibility of false positive results Genomic DNA from T. equigenitalis (Type strain NCTC 11184 and two wild-type strains isolated previously from horses with CEM), Pseudomonas aeruginosa, Alcaligenes xylosoxidans, Haemophilus influenzae, Stenotrophomonas maltophilia, Burkholderia cepacia, CDC Group IV organisms, Streptococcus pneumoniae and Staphylococcus aureus was extracted from pure culture employing the Roche High Purity PCR DNA extraction kit (Roche Ltd., England), as per the manufacturer's instructions.

\section{PCR amplification}

Amplification reactions were set-up in accordance with Good Molecular Diagnostic Procedures, as detailed in the guidelines of Millar et al. (2002). In order to minimise contamination, all reaction mixes were set up in a PCR hood in a room separate from that used to extract DNA and from the amplification and post- PCR room. Initially, PCR amplification conditions were optimised by separately varying magnesium chloride concentration, annealing temperature, primer concentration and DNA template concentration. Following optimisation, reaction mixes $(25 \mu \mathrm{l})$ were set up as follows: $-10 \mathrm{mM}$ Tris- $\mathrm{HCl}, \mathrm{pH} 8.3,50 \mathrm{mM} \mathrm{KCl}, 2.5 \mathrm{mM}$ $\mathrm{MgCl} 2,200 \mu \mathrm{M}$ (each) dATP, dCTP, dGTP and dTTP; 1.25U of
Taq DNA polymerase (Amplitaq; Perkin Elmer), 0 . $1 \mu \mathrm{M}$ (each) of each primers (TE16SrRNABCHf and TEl6SrRNABCH; F i g u re $\mathbf{l}$ ) and $2 \mu \mathrm{l}$ of DNA template. Following a "hot start", the reaction mixtures were subjected to the following empirically optimised thermal cycling parameters in a Perkin Elmer 2400 thermocycler: $96^{\circ} \mathrm{C}$ for five minutes followed by 40 cycles of twostep PCR; $96^{\circ} \mathrm{C}$ for one minute, $72^{\circ} \mathrm{C}$ for one minute, followed by a final extension at $72^{\circ} \mathrm{C}$ for 10 minutes. During each run, molecular grade water was included randomly as negative controls and appropriate DNA templates from T. equigenitalis DNA were included as positive controls, as appropriate.

\section{Detection of PCR products}

Following amplification, aliquots $(15 \mu \mathrm{l})$ were removed from each reaction mixture and examined by electrophoresis $(80 \mathrm{~V}, 45$ minutes) in gels composed of $2 \%(\mathrm{w} / \mathrm{v})$ agarose (Gibco, UK) in TAE buffer (40mM Tris, $20 \mathrm{mM}$ acetic acid, ImM EDTA, pH 8.3), stained with ethidium bromide $(5 \mu \mathrm{g} / 100 \mathrm{ml})$. Gels were visualised under UV illumination using a gel image analysis system (UVP Products, England) and all images archived as digital graphic files (*.jpeg).

\section{Surveillance study of Thoroughbred horses}

Two hundred and fifty Thoroughbred horses were examined in this study for the presence of T. equigenitalis by PCR and cultural techniques. One swab was taken from each animal by a veterinary surgeon. In mares, the swab was taken from either the clitoral fossa or the clitoral sinus and in stallions, from the urethral orifice, the

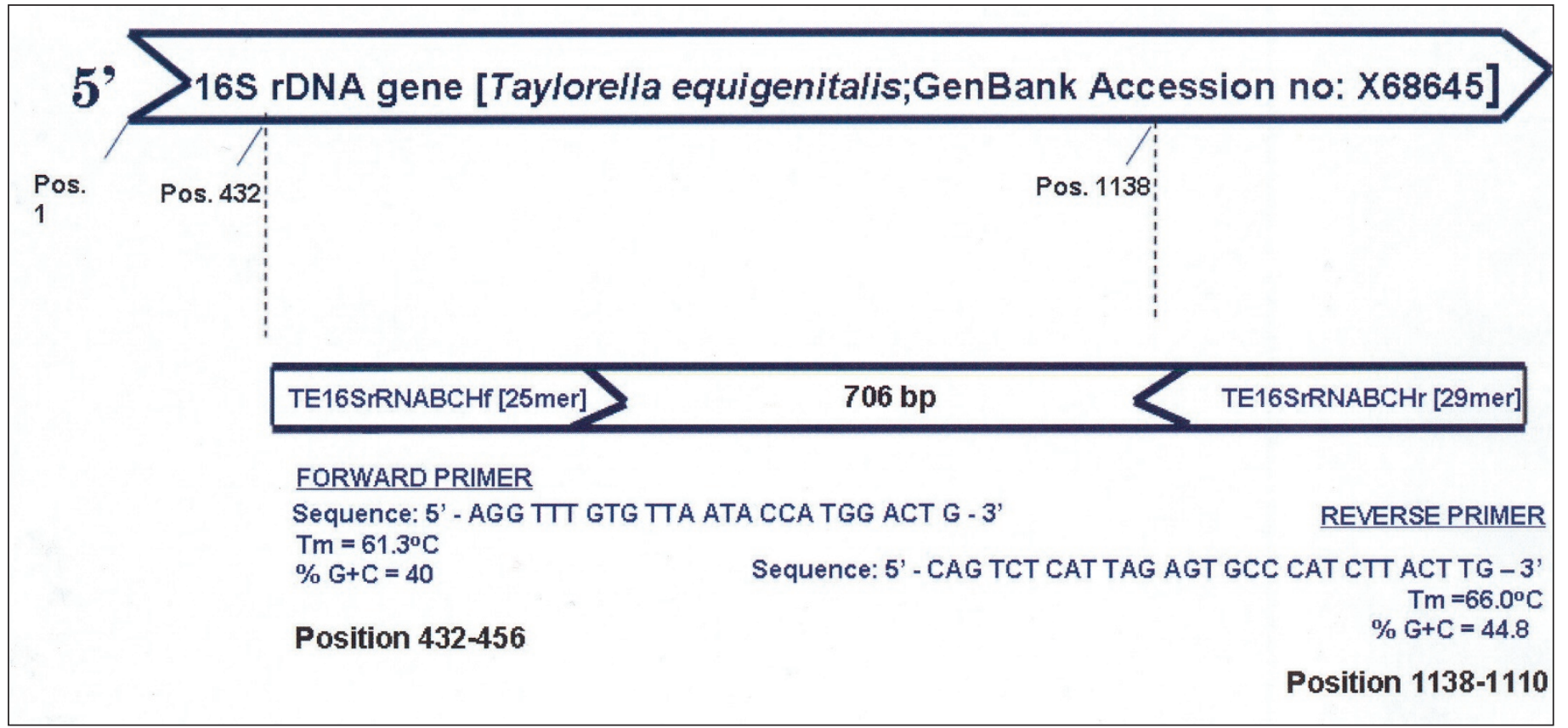

FIGURE I: Description and location of novel PCR primers employed in the PCR assay. 
urethral fossa or the penile sheath. Swabs were transported to the laboratory in Amies charcoal transport media and were examined within 24h. For the conventional detection of T. equigenitalis, swabs were plated on to four different media:

(i) CEMO agar (MAST DM470, MAST Diagnostics Ltd, Merseyside, England) containing streptomycin $(250 \mu \mathrm{g} / \mathrm{ml})$ and fungizone $(5 \mu \mathrm{g} / \mathrm{ml})$;

(ii) CEMO agar supplemented with $5 \%$ chocolate and fungizone $(5 \mu \mathrm{g} / \mathrm{ml})$

(iii) Columbia agar base supplemented with 5\% defibrinated horse blood; and,

(iv) McConkey agar. The plates were incubated for six days $\left(37^{\circ} \mathrm{C} ; 5 \%[\mathrm{v} / \mathrm{v}] \mathrm{CO} 2\right)$.

Swabs from animals were placed in $1.5 \mathrm{ml}$ micro-centrifugation

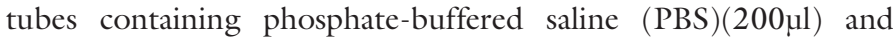
sonicated in a sonicating bath for five minutes. The resulting suspension was boiled for 10 minutes in a heating block (Stuart Scientific Ltd., England) and subsequently centrifuged at $13,000 \mathrm{~g}$ for five minutes. A $100 \mu \mathrm{l}$ volume of supernatant was transferred to a tube and stored at $-80^{\circ} \mathrm{C}$ until amplification. Following this, the pellet was re-suspended in sterile distilled water $(50 \mathrm{ml})$ and boiled for 15 minutes.

A sterile non-inoculated swab and a swab spiked with T. equigenitalis (Type strain NCTC 11184) underwent the above extraction procedure to serve, respectively, as a negative and a positive extraction control. The sensitivity of the assay was calculated by serially diluting a known number of bacterial cells in PBS and carrying out an extraction and PCR amplification, as described above. The highest serial dilution that yielded a positive PCR reaction was considered to be the threshold of sensitivity for the assay.

\section{Results}

Two primers [TE16SrRNABCH forward $_{\text {and TE16SrRNABCH }}$ reverse were selected from the $16 \mathrm{~S}$ rRNA gene of $\mathrm{T}$. equigenitalis as outlined in Figure 1. We tested the specificity of the primers both by BLAST searching at http://www.blast.genome.ad.jp, as well as by challenging the primers to DNA from non-T. equigenitalis organisms. BLAST searching demonstrated a score of 50 [E value 6x10-5] and 49 [E value 2x10-4] for the forward and reverse primers, respectively. There was no shared homology with any other bacterial or eukaryotic DNA with either primer, nor with the other species member within the genus Taylorella (i.e., T. asinigenitalis) where there were eight and four nonaligning base pairs in the forward and reverse primers, respectively, between these two species. An amplified product of approximately 706bp was generated with all tested T. equigenitalis isolates (Figure 2) and no other bacterial genomic DNA was amplified, demonstrating the specificity of the primers for $\mathrm{T}$. equigenitalis. Of the 250 animals screened for T. equigenitalis, none was positive for this organism by either culture or PCR methods, where the molecular sensitivity of the assay was shown to be no lower than 36 cells, which compares favourably to the three-step PCR assay, previously described by Bleumink-Pluym et al. (1994), who calculated the sensitivity of their assay as 15 bacteria.

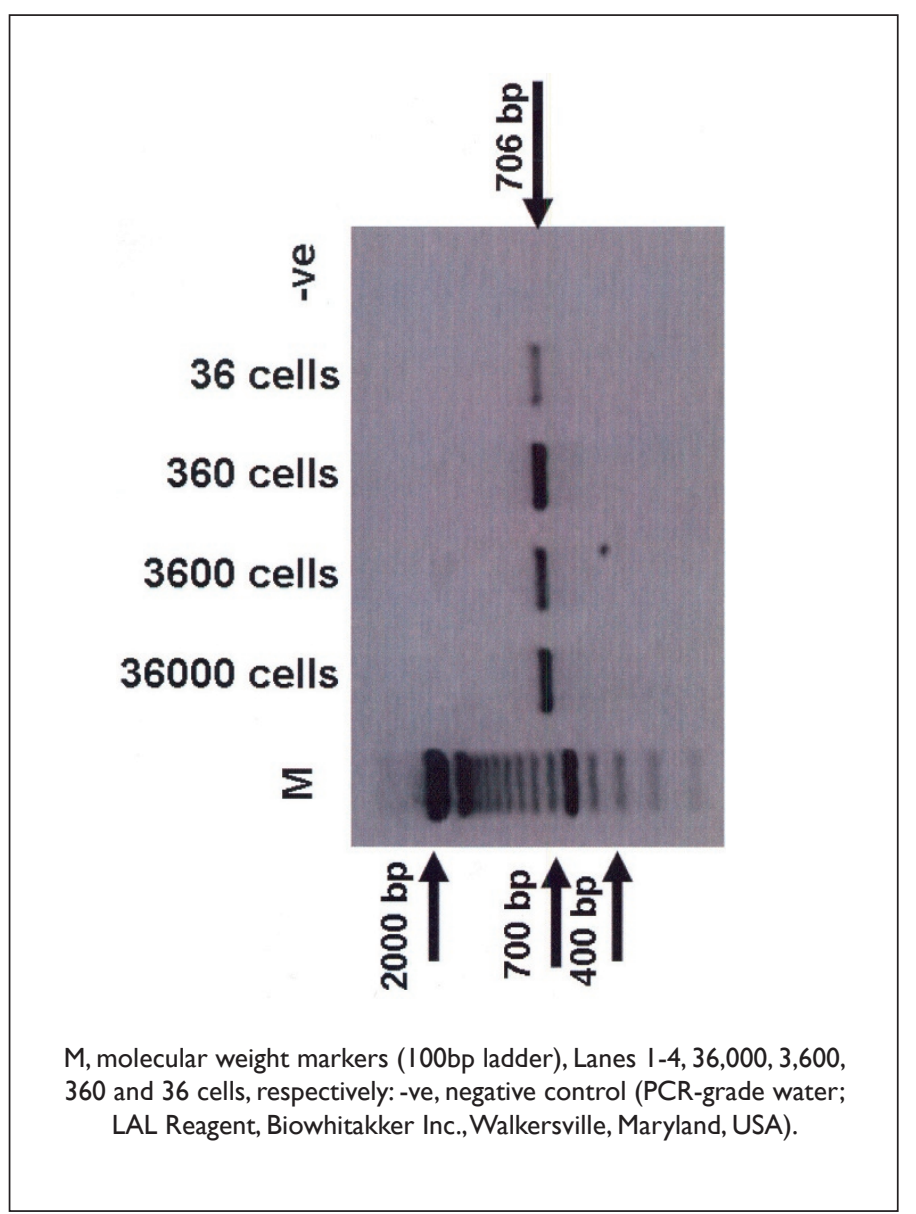

FIGURE 2: Sensitivity of the two-step PCR assay; PCR amplification of a 706bp region of the I6S rDNA of Taylorella equigenitalis

\section{Discussion}

The traditional basis for the identification of pathogenic and commensal bacteria has been their isolation or propagation in the laboratory. Biochemical, morphological and serological tests usually require growth of the organism. Reliance on these parameters may have significantly limited the awareness of true bacterial diversity and is impractical in many situations. The rapidly expanding use of $16 \mathrm{~S}$ rRNA gene sequences for phylogenetic, evolutionary and diagnostic studies offers an opportunity for alternative approaches: $16 \mathrm{~S}$ rRNA genes are found in all bacteria and accumulate mutations at a slow, constant rate over time, hence they may be used as "molecular clocks" (Woese, 1987). Highly variable portions of the 16S rRNA sequence provide unique signatures to any bacterium and useful information about relationships between them. Alternatively, since 16S rRNA molecules have crucial structural constraints, certain conserved regions of sequence are found in all known bacteria (i.e., the eubacteria) and in all known organisms. Species-specific primers may then be designed to recognise these conserved bacterial 16S rRNA gene sequences and used to amplify 
intervening, variable or diagnostic regions (Weisburg et al., 1991; Wilson et al., 1990). This procedure avoids the need to grow the bacterium and does not require pre-existing phylogenetic information. Employment of conventional culture techniques for the detection of $\mathrm{T}$. equigenitalis can be both slow and insensitive, potentially leading to the generation of false-negative results. In the case of $T$. equigenitalis, normally six days are required for the detection by culture (Swaney and Sahu, 1978). Phenotypic identification can be further compromised due to the presence of unusual colony morphology variants (Sahu et al., 1982) and streptomycinsensitivity variants (Kamada et al., 1986). Recently, there is an increasing tendency to employ PCR-based detection assays for a number of clinically relevant pathogens. Although such a molecular approach may have certain disadvantages, overall the employment of PCR-based detection assays has numerous advantages, which outweigh those offered by the conventional approach: in part icular, improvements in the speed and specificity of the detection of T. equigenitalis, as well as the ability to detect this organism in animals underg o in $\mathrm{g}$ antimicrobial chemotherapy. Disease prevention strategies rely heavily on the results of swabs taken from mares and stallions involved in breeding programmes. Sole reliance on problematical culture techniques may translate into complications in the management of horses at stud, e.g., where stallion owners should not accept mares unless they have an uptodate negative laboratory certificate (Anon, 2000). Conventionally, most PCR assays involve a three-step process, i.e., denaturation, annealing and extension. However, this method sought to develop a novel two-step process, i.e., denaturation and annealing/extension, to gain on time of phenotypic identification. Consequently, large $25+$ mers were designed to be able to tolerate such a two-step PCR assay. Overall, it was estimated that the two-step assay would gain approximately one hour in comparison with the three-step assay, allowing for 40 cycles of one minute annealing at approximately $50^{\circ} \mathrm{C}$ to $60^{\circ} \mathrm{C}$, in addition to the ramp times in lowering the temperature from denaturation to annealing and from annealing to extension. This advantage in saving time may be beneficial in busy service diagnostic laboratories, to help facilitate several PCR runs within the working day. Although the ribosomal primers that were designed in this study are specific for $\mathrm{T}$. equigenitalis, it must be remembered that the $6 \mathrm{~S}$ rRNA gene is highly conserved throughout the eubacteria. Consequently, if a positive swab is detected by PCR, it must be regarded as being presumptively positive, until confirmed by sequencing of the 706bp PCR amplicon. As positive results are extremely rare, it is indeed feasible to sequence all presumptive positives to avoid any molecular misidentifications. In conclusion, we describe a novel two-step PCR assay for the detection of T. equigenitalis based on specific primers within the 16S rRNA genes. Such a molecular approach offers a sensitive and specific alternative to conventional culture techniques, which could potentially lead to improved diagnosis and subsequent management of horses involved in breeding programmes.

\section{Acknowledgements}

Paula Gibson was funded by a Wellcome Vacation Studentship.

\section{References}

Anon. (2000). Abbreviated codes of practice for the control of equine diseases for the 2000-2003 breeding seasons. Contagious equine metritis, other equine bacterial venereal diseases, Equine viral arteritis. Dublin: Department of Agriculture, Food and Rural Development.

Bleumink-Pluym, N.M.C., Werdler, M.E.B., Houwers, D.J.,
Parlevliet, J.M., Colenbrander, B. and van der Zeijst, B.A.M. (1994). Development and evaluation of PCR test for detection of Taylorella equigenitalis. Journal of Clinical Microbiology 32: 893896.

Crowhurst, R.C. (1977). Genital infection in mares. Veterinary Record 100: 476.

Kamada, M., Kumanomido, T., Anzai, T., Kanemaru, T., Senba, H. and Ohishi, H. (1986). Isolation and drug susceptibility of streptomycin sensitive Taylorella equigenitalis from mares with metritis and infertility in Japan. Bulletin Equine Research Institute 24: 23-32.

Millar, B.C., Xu, J. and Moore, J.E. (2002). Risk assessment models and contamination management: implications for broadrange ribosomal DNA PCR as a diagnostic tool in medical bacteriology. Journal of Clinical Microbiology 40: 1575-1580. Sahu, S.P., Wool, S. and Breese, S.S. Jr. (1982). Observation on the morphology of contagious equine metritis bacterial colonies isolated from infected pony mares. American Journal of Veterinary Research 43: 796-800.

Swaney, L.M. and Sahu, S.P. (1978). CEM: Bacteriological methods. Veterinary Record 102: 43.

Timoney, P.J. (1996). Contagious equine metritis. Comparative Immunology, Microbiology and Infectious Diseases 19: 199-204.

Weisburg, W.G., Barns, S.M., Pelletier, D.A. and Lane, D.J. (1991). 16S ribosomal DNA amplification for phylogenetic study. Journal of Bacteriology 173: 697-703.

Widjojoatmodjo, M.N., Fluit, A.C. and Verhoef, J. (1994). Rapid identification of bacteria by PCR-single-strand conformation polymorphism. Journal of Clinical Microbiology 32: 3002-3007.

Wilson, K.H., Blitchington, R.B. and Greene, R.C. (1990). Amplification of bacterial 165 ribosomal DNA with polymerase chain reaction. Journal of Clinical Microbiology 28: 1942-1946.

Woese, C.R. (1987). Bacterial evolution. Microbiological Reviews 51: 221-271. 